\title{
Using Split-Ring Resonators to Measure Complex Permittivity and Permeability
}

\author{
J.S. Bobowski \\ Department of Physics, University of British Columbia Okanagan, \\ 3333 University Way, Kelowna, BC, V1V 1V7, Canada
}

(Dated: August 24, 2015)

\begin{abstract}
This paper describes how to measure the complex permittivity and permeability of materials using split-ring resonators. High- $Q$ split-ring resonators, which can be modelled as $L R C$ circuits, are easy to fabricate and can be used to measure electromagnetic material properties at frequencies that span approximately $10 \mathrm{MHz}$ to $2 \mathrm{GHz}$. If the resonator is submerged in a liquid/suspension, its resonant frequency and quality factor will be be modified from the in-air values by factors that depend on the relative permittivity and permeability of the liquid/suspension. General expressions for the resonator's frequency response are derived. Unlike an $L R C$ circuit, the resonator's response is not strictly Lorentzian. However, a wide variety of cases for which the response is approximately Lorentzian are explored. For each of these cases it is demonstrated that the real and imaginary components of the relative permittivity and permeability can be extracted from the in-air and in-liquid/suspension resonant frequencies and quality factors.
\end{abstract}

Keywords: Split-ring resonator, Permittivity, Permeability, Quality factor

PACS numbers: 01.50.Pa, 77.22.Ch, 75.30.Cr

\section{INTRODUCTION}

Split-ring resonators (SRRs) enjoy a wide variety of uses in current experimental physics research. They are key components of engineered metamaterials ${ }^{\mathbf{m}}$, used as tools for measuring the electromagnetic (EM) properties of materials ${ }^{\mathbb{\square}}$, and used as devices in novel microwave

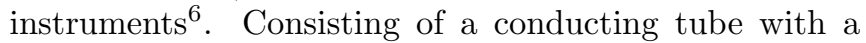
slit along its length (Fig. $\mathbf{d}$ ), SRRs that operate from approximately 10 to $2000 \mathrm{MHz}$ are easy to fabricate $\mathbf{\square [}$.

This paper describes how SRRs can be used to measure both the real and imaginary components of the permittivity and permeability of materials or suspensions. This work is an extension of Ref. $\mathbf{Q}$ in which a SRR was used to measure the dielectric constant and conductivity of saltwater solutions with varying concentrations of salt.

The paper is organized as follows: Section $\mathbf{\square}$ reviews the properties of SRRs when suspended in air. Section $\mathbb{W}$ examines how the SRR properties are modified when submerged in a liquid with a complex permittivity. It is shown that, under many circumstances, the $\mathrm{SRR}$ response is approximately Lorentzian. Several real materials are consider to demonstrate how, in principle, the real and imaginary components of the permittivity can be extracted from successive measurements of the in-air and in-liquid resonant frequencies and quality factors. Section $\mathbb{\nabla}$ considers the case of a SRR submerged in a suspension of magnetic particles and shows how the real and imaginary components of the permeability can be extracted. The results are summarized in Sec. $\nabla$.

\section{SUSPENDED IN AIR}

A split-ring resonator can be modelled as an $L R C$ series circuit ${ }^{\mathrm{D}}$. The slit is modelled as a capacitor with $C_{0} \approx \varepsilon_{0} w \ell / t$ and the bore as an inductor with $L_{0} \approx \mu_{0} \pi r_{0}^{2} / \ell$. The resulting resonance frequency is (a)

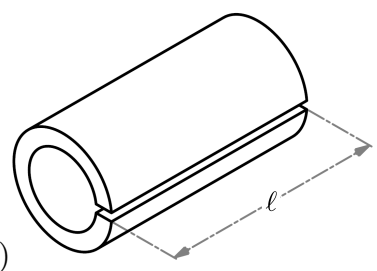

(b)

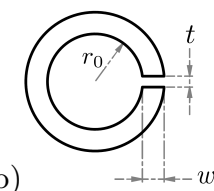

FIG. 1. (a) A schematic drawing of a SRR. (b) Cross-sectional view. The SRR has length $\ell$, inner radius $r_{0}$, wall thickness $w$, and a slit along its length of height $t$. In this work, dimensions that match the SRR reported in Ref. $\mathbf{\square}$ were used in all calculations. Namely, $\ell=4$ in. $(10.2 \mathrm{~cm}), r_{0}=11 / 16$ in. $(1.75 \mathrm{~cm})$, $w=5 / 16$ in. $(0.79 \mathrm{~cm})$, and $t=0.010 \mathrm{in}$. $(0.25 \mathrm{~mm})$.

given by

$$
\omega_{0}=\frac{1}{\sqrt{L_{0} C_{0}}} \approx \frac{c}{r_{0}} \sqrt{\frac{t}{\pi w}}
$$

where $c=1 / \sqrt{\varepsilon_{0} \mu_{0}}$ is the speed of light.

The resistance of the SRR determines the width of the resonance. Currents are restricted to be within a length $\delta=\sqrt{2 \rho /\left(\mu_{0} \omega\right)}$ of the inner surface of the SRR bore where $\delta$ is the EM skin depth. As a result, the effective resistance of SRR is given by

$$
R(\omega)=\rho \frac{2 \pi r_{0}}{\ell \delta(\omega)} \propto \sqrt{\omega}
$$

where the explicit dependence on frequency has been indicated for later use. For a SRR made from a good conductor, $\omega_{0}$ is expected to be much greater than the width of the resonance such that, over the frequency range of interest, the SRR resistance can be approximated as $R\left(\omega_{0}\right) \equiv R_{0}$ which leads to a simple expression for the expected quality factor $Q_{0}^{-1}=R_{0} \sqrt{C_{0} / L_{0}}=\delta / r_{0}$. An aluminum SRR made using the dimensions given in Fig. $\square$ results in $Q_{0}>3000$ which justifies approximating $R(\omega)$ 
as $R_{0}$. In practice, because the SRR experiences radiative losses, quality factors that approach the value predicted by $Q_{0}$ are achieved only if the resonator is surrounded by a larger cylindrical EM shield $\mathbf{D}^{\mathbf{D}}$.

The detected SRR signal is proportional to the magnitude of the induced current and, therefore, inversely proportional to the magnitude of the effective impedance $Z_{0}$ which has a Lorentzian line shape given by:

$$
\begin{aligned}
\left|Z_{0}(\omega)\right| & =\left[R_{0}^{2}+\left(\omega L_{0}-\frac{1}{\omega C_{0}}\right)^{2}\right]^{1 / 2} \\
& =R_{0} Q_{0}\left[\frac{1}{Q_{0}^{2}}+\left(\frac{\omega}{\omega_{0}}-\frac{\omega_{0}}{\omega}\right)^{2}\right]^{1 / 2}
\end{aligned}
$$

where the second equation has been obtained using the substitutions $C_{0}^{-1}=\omega_{0} R_{0} Q_{0}$ and $L_{0}=R_{0} Q_{0} / \omega_{0}$. Fits to the frequency dependence of $\left|Z_{0}(\omega)\right|^{-1}$ allow $\omega_{0}$ and $Q_{0}$ to be extracted from the measured in-air SRR signal ${ }^{\mathbf{g}}$.

\section{RELATIVE PERMITTIVITY}

Now imagine submerging the SRR in a liquid having a relative permittivity $\varepsilon_{\mathrm{r}}=\varepsilon^{\prime}-j \varepsilon^{\prime \prime}$. The liquid fills the gap of the SRR where the electric field is concentrated resulting in an enhanced and complex effective capacitance $C_{1}=\varepsilon_{\mathrm{r}} C_{0}$ and a modified resonance frequency $\omega_{1}$. The inductance is unaffected by the permittivity. However, recall from Eq. $\nabla$ that the SRR resistance is proportional to $\sqrt{\omega}$ such that the SRR impedance becomes:

$$
\begin{aligned}
& Z_{1}(\omega)=R_{0} \sqrt{\frac{\omega}{\omega_{0}}}+j \omega L_{0}+\frac{1}{j \omega \varepsilon_{\mathrm{r}}(\omega) C_{0}} \\
& =\left[R_{0} \sqrt{\frac{\omega}{\omega_{0}}}+\frac{\varepsilon^{\prime \prime}}{\left|\varepsilon_{\mathrm{r}}\right|^{2}} \frac{1}{\omega C_{0}}\right]+j\left[\omega L_{0}-\frac{\varepsilon^{\prime}}{\left|\varepsilon_{\mathrm{r}}\right|^{2}} \frac{1}{\omega C_{0}}\right]
\end{aligned}
$$

where $\left|\varepsilon_{\mathrm{r}}\right|^{2} \equiv\left(\varepsilon^{\prime}\right)^{2}+\left(\varepsilon^{\prime \prime}\right)^{2}$. Notice that a complex $\varepsilon_{\mathrm{r}}$ leads to an additional real term in $Z_{1}$ and hence additional losses. If $C_{0}$ and $L_{0}$ are once again eliminated in favour of $\omega_{0}$ and $Q_{0}$ the result is:

$$
\begin{aligned}
\frac{\left|Z_{1}(\omega)\right|}{R_{0} Q_{0}}=\{ & {\left[\frac{1}{Q_{0}} \sqrt{\frac{\omega}{\omega_{0}}}+\frac{\varepsilon^{\prime \prime}(\omega)}{\left|\varepsilon_{\mathrm{r}}(\omega)\right|^{2}} \frac{\omega_{0}}{\omega}\right]^{2} } \\
+ & {\left.\left[\frac{\omega}{\omega_{0}}-\frac{\varepsilon^{\prime}(\omega)}{\left|\varepsilon_{\mathrm{r}}(\omega)\right|^{2}} \frac{\omega_{0}}{\omega}\right]^{2}\right\}^{1 / 2} }
\end{aligned}
$$

where the fact that $\varepsilon_{\mathrm{r}}$ is frequency dependent has been explicitly indicated. Although the general result given by Eq. $\square$ exhibits resonant behaviour, it is non-Lorentzian because $\Re\left[Z_{1}\right]$ is not frequency independent and because of the $\varepsilon_{\mathrm{r}}(\omega)$ frequency dependence in $\Im\left[Z_{1}\right]$. The SRR response can be fit to the frequency dependence of $\left|Z_{1}(\omega)\right|^{-1}$ to extract experimental values of $\varepsilon^{\prime}$ and $\varepsilon^{\prime \prime}$ near the resonance frequency $\omega_{1}$.

Alternatively, if the width of the resonance is much less than $\omega_{1}$ and $\varepsilon_{\mathrm{r}}(\omega)$ does not change too rapidly, the

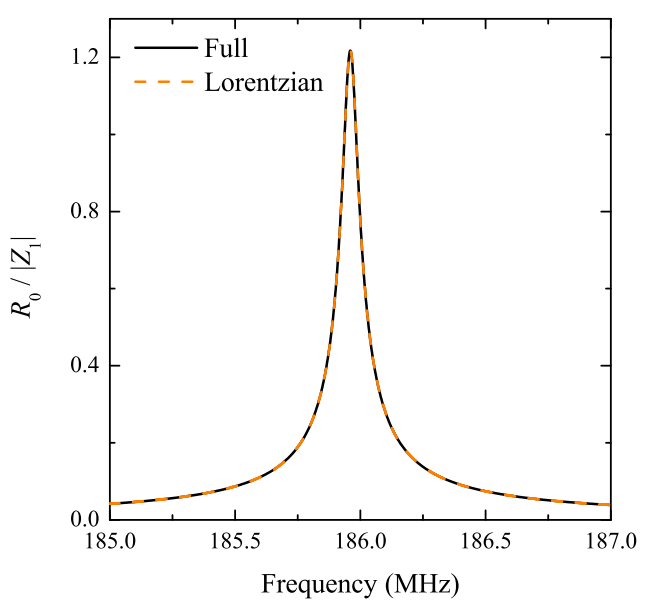

FIG. 2. Comparison of the exact $R_{0} /\left|Z_{1}\right|$ (Eq. $\mathbf{0}$, solid line) to the approximate Lorentzian curve (dashed line) with Teflon $\left(\varepsilon_{\mathrm{r}}=2.2\right)$ filling the gap of the SRR. All calculations done assuming an aluminum $\left(\rho=2.82 \times 10^{-8} \Omega^{-1} \mathrm{~m}^{-1}\right)$ SRR made using the dimensions given in Fig. $\mathrm{W}$.

shape of the SRR response is approximately Lorentzian with $\omega_{1}$ and the quality factor determined from:

$$
\begin{aligned}
\omega_{1} & \approx \frac{\sqrt{\varepsilon^{\prime}\left(\omega_{1}\right)}}{\left|\varepsilon_{\mathrm{r}}\left(\omega_{1}\right)\right|} \omega_{0} \\
\frac{1}{Q_{1}} & \approx \frac{1}{Q_{0}}\left[\frac{\left|\varepsilon_{\mathrm{r}}\left(\omega_{1}\right)\right|^{2}}{\varepsilon^{\prime}\left(\omega_{1}\right)}\right]^{1 / 4}+\frac{\varepsilon^{\prime \prime}\left(\omega_{1}\right)}{\varepsilon^{\prime}\left(\omega_{1}\right)} .
\end{aligned}
$$

The following sections show how the SRR can be used to extract $\varepsilon^{\prime}$ and $\varepsilon^{\prime \prime}$ from various materials. Three cases are explored: A) the $Q_{0}^{-1}$ term in Eq. Q 0 dominates, B) the $\varepsilon^{\prime \prime}$ term dominates, and $\mathrm{C}$ ) the two terms are comparable.

\section{A. $Q_{0}^{-1}$ term dominant}

If the $Q_{0}^{-1}$ term in Eq. $\mathbb{Q}$ is dominant, then $\varepsilon^{\prime \prime}$ is negligible and $\varepsilon_{\mathrm{r}} \approx \varepsilon^{\prime}$ such that $\omega_{1} \approx \omega_{0} / \sqrt{\varepsilon^{\prime}}$ and $Q_{1} \approx Q_{0} \sqrt[4]{\varepsilon^{\prime}}$. As an example, consider inserting a tight-fitting piece of Teflon, for which $\varepsilon_{\mathrm{r}}=2.2$, into the gap of the SRR. Figure $\nabla$ compares the exact $R_{0} /\left|Z_{1}\right|$ calculated using Eq. $\square$ to the Lorentzian approximation obtained by replacing $\omega_{0}$ and $Q_{0}$ in Eq. $\nabla$ by the $\omega_{1}$ and $Q_{1}$ expressions given above and in the first row of Table $\mathbb{m}$. The approximate Lorentzian is indistinguishable from the exact curve and, as shown in Table $\mathbb{W}$, experimental values of $\varepsilon^{\prime}$ can be determined from either $\left(\omega_{0} / \omega_{1}\right)^{2}$ or $\left(Q_{0} / Q_{1}\right)^{4}$.

\section{B. $\varepsilon^{\prime \prime}$ term dominant}

The opposite extreme occurs when the $\varepsilon^{\prime \prime}$ term dom-

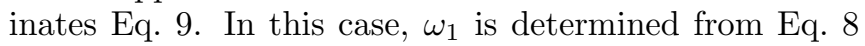
and $Q_{1} \approx \varepsilon^{\prime} / \varepsilon^{\prime \prime}$. To numerically solve Eq. $\boldsymbol{\nabla}$ for $\omega_{1}$, the 


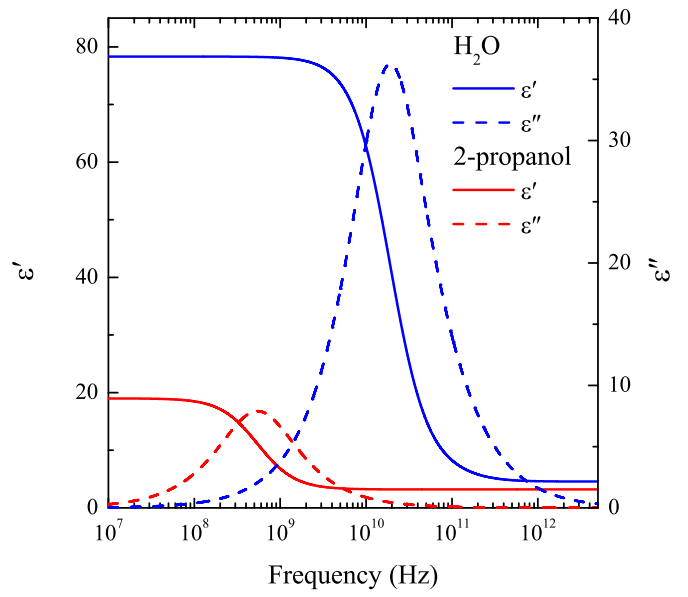

FIG. 3. The complex relative permittivity of 2-propanol $\mathbf{l}^{\mathbf{g}}$ and water as a function of frequency.

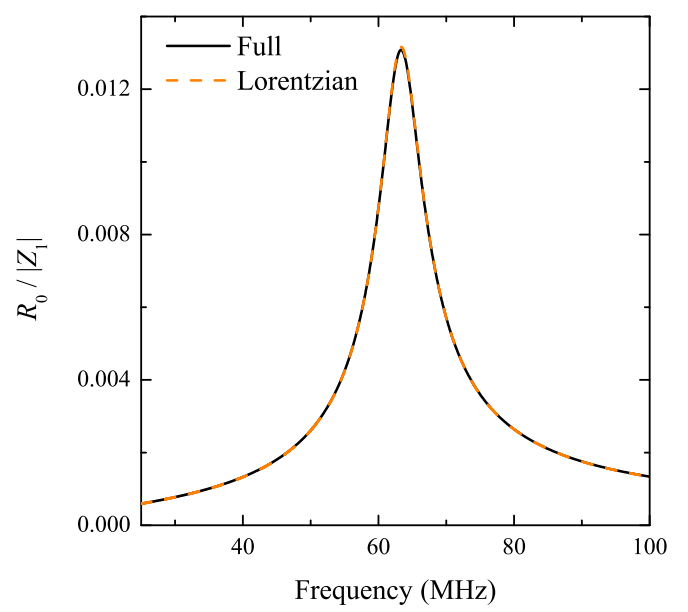

FIG. 4. Comparison of the exact $R_{0} /\left|Z_{1}\right|$ (Eq. $\square$, solid line) to the approximate Lorentzian curve (dashed line) with the SRR submerged in 2-propanol.

frequency dependencies of the real and imaginary components of $\varepsilon_{\mathrm{r}}$ must be known. As an example, Fig. (3 shows the relative permittivity of 2 -propanol ${ }^{\mathbf{\mathbb { }}}$ as a function of frequency ${ }^{\mathbb{W}}$. With these assumed frequency dependencies, Eq. $\mathbf{\nabla}$ yields $\omega_{1} /(2 \pi)=63.3 \mathrm{MHz}$ and $Q_{1}^{-1} \approx 0.1 \gg Q_{0}^{-1}$. Figure $\mathbb{\theta}$ shows $R_{0} /\left|Z_{1}\right|$ calculated using the exact expression given by Eq. $\square$ and the approximate Lorentzian curve obtained using the $\omega_{1}$ and $Q_{1}$ expressions in the second row of Table $\mathbb{W}$. Once again, the exact and approximate curves are, for all practical purposes, indistin-

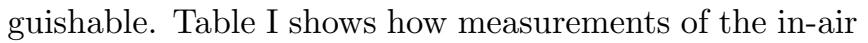
and in-liquid resonance frequencies and quality factors can be used to experimentally determine $\varepsilon^{\prime}$ and $\varepsilon^{\prime \prime}$.

\section{C. $Q_{0}^{-1} \& \varepsilon^{\prime \prime}$ terms comparable}

Finally, water is used as an example of a material for which the $Q_{0}^{-1}$ and $\varepsilon^{\prime \prime}$ terms are comparable. The permit-

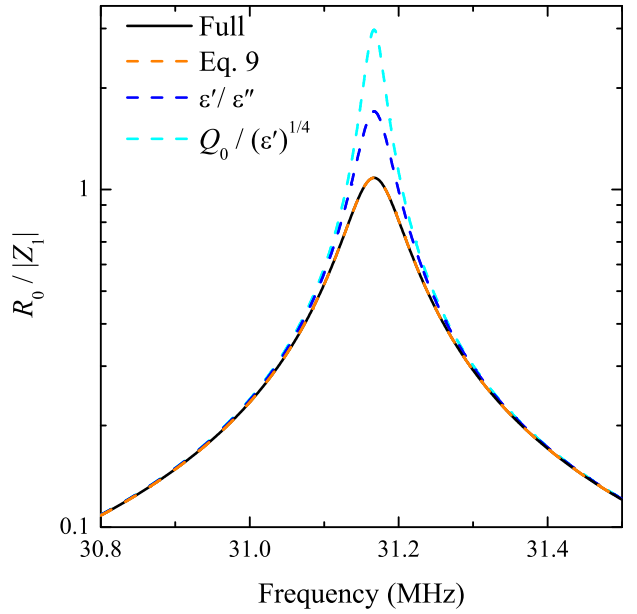

FIG. 5. Comparison of the exact $R_{0} /\left|Z_{1}\right|$ (Eq. $\square$, solid line) to the Lorentzian curves (dashed lines) with the SRR submerged in water. The three Lorentzians were generated using $Q_{1}=Q_{0}\left(\varepsilon^{\prime}\right)^{-1 / 4}, Q_{1}=\varepsilon^{\prime} / \varepsilon^{\prime \prime}$, and $Q_{1}$ given by Eq. 0 . The vertical axis is shown on a log scale to emphasize the differences between the three Lorentzian curves.

tivity of water is shown in Fig. $\mathrm{B}^{\mathrm{m}}$. The resonance frequency obtained from Eq. $\nabla$ is $\omega_{1} /(2 \pi)=31.2 \mathrm{MHz}$. Figure 5 compares the exact form of $R_{0} /\left|Z_{1}\right|$ to Lorentzian curves obtained using $Q_{1}=Q_{0}\left(\varepsilon^{\prime}\right)^{-1 / 4}, Q_{1}=\varepsilon^{\prime} / \varepsilon^{\prime \prime}$, and $Q_{1}$ as given in Eq. [. In this case, the full expression for $Q_{1}$ is required to accurately reproduce the frequency dependence of $\left|Z_{1}\right|^{-1}$. Table $\mathbb{\text { I }}$ shows how the real and imaginary components of $\varepsilon_{\mathrm{r}}$ can be extracted from in-air and in-liquid measurements of the SRR resonance frequency and quality factor.

\section{RELATIVE PERMEABILITY}

The same type of analysis presented in Sec. $\mathbf{W}$ can be repeated for the case of a SRR submerged in a material having a relative permeability $\mu_{\mathrm{r}}=\mu^{\prime}-j \mu^{\prime \prime}$. In this case, the inductance $L_{2}=\mu_{\mathrm{r}} L_{0}$ is modified such that:

$$
\frac{\left|Z_{2}(\omega)\right|}{R_{0} Q_{0}}=\left\{\left[\frac{1}{Q_{0}} \sqrt{\frac{\omega}{\omega_{0}}}+\mu^{\prime \prime} \frac{\omega}{\omega_{0}}\right]^{2}+\left[\mu^{\prime} \frac{\omega}{\omega_{0}}-\frac{\omega_{0}}{\omega}\right]^{2}\right\}^{1 / 2}
$$

which, again, is a non-Lorentzian response. Of course, if the modified resonance frequency $\omega_{2}$ is large compared to the width of the resonance, an approximately Lorentzian response is recovered. The relationships between $\omega_{2}, Q_{2}$, $\mu^{\prime}$, and $\mu^{\prime \prime}$ for the various cases $\left(Q_{0}^{-1}\right.$ dominant, $\mu^{\prime \prime}$ dominant, and the general case in which the two terms are comparable) are summarized in the bottom half of Table $\mathbf{m}$.

Because of its geometry, it is only practical to use SRRs to measure the permeability of magnetic particles suspended in a liquid. One would first measure the resonance of the SRR submerged in the liquid without any 


\begin{tabular}{|c|c|c|c|c|}
\hline Permittivity & $\omega_{1}$ & $Q_{1}$ & $\varepsilon^{\prime}\left(\omega_{1}\right)$ & $\varepsilon^{\prime \prime}\left(\omega_{1}\right)$ \\
\hline $\begin{array}{c}Q_{0}^{-1} \\
\text { dominant }\end{array}$ & $\frac{\omega_{0}}{\sqrt{\varepsilon^{\prime}}}$ & $\frac{Q_{0}}{\left(\varepsilon^{\prime}\right)^{1 / 4}}$ & $\left(\frac{\omega_{0}}{\omega_{1}}\right)^{2}$ and $\left(\frac{Q_{0}}{Q_{1}}\right)^{4}$ & - \\
\hline $\begin{array}{c}\varepsilon^{\prime \prime} \\
\text { dominant }\end{array}$ & $\omega_{0} \frac{\sqrt{\varepsilon^{\prime}}}{\left|\varepsilon_{\mathrm{r}}\right|}$ & $\frac{\varepsilon^{\prime}}{\varepsilon^{\prime \prime}}$ & $\frac{\left(Q_{1} \frac{\omega_{0}}{\omega_{1}}\right)^{2}}{1+Q_{1}^{2}}$ & $\frac{Q_{1}\left(\frac{\omega_{0}}{\omega_{1}}\right)^{2}}{1+Q_{1}^{2}}$ \\
\hline general & $\omega_{0} \frac{\sqrt{\varepsilon^{\prime}}}{{ }^{\prime}}$ & {$\left[1,\left(\underline{\left|\varepsilon_{\mathrm{r}}\right|^{2}}\right)^{1 / 4}+\underline{\varepsilon^{\prime \prime}}\right]$} & $\left(\frac{\omega_{0}}{\omega_{1}}\right)^{2}$ & $\left(\frac{\omega_{0}}{\omega_{1}}\right)^{2}\left[\frac{1}{Q_{1}}-\frac{1}{Q_{0}}\left(\frac{\omega_{0}}{\omega_{1}}\right)^{1 / 2}\right]$ \\
\hline general & $\omega_{0} \overline{\left|\varepsilon_{\mathrm{r}}\right|}$ & $\left.\overline{Q_{0}}\left(\overline{\varepsilon^{\prime}}\right)+\overline{\varepsilon^{\prime}}\right]$ & $1+\left[\frac{1}{Q_{1}}-\frac{1}{Q_{0}}\left(\frac{\omega_{0}}{\omega_{1}}\right)^{1 / 2}\right]$ & $1+\left[\frac{1}{Q_{1}}-\frac{1}{Q_{0}}\left(\frac{\omega_{0}}{\omega_{1}}\right)^{1 / 2}\right]$ \\
\hline Permeability & $\omega_{2}$ & $Q_{2}$ & $\mu^{\prime}\left(\omega_{2}\right)$ & $\mu^{\prime \prime}\left(\omega_{2}\right)$ \\
\hline $\begin{array}{c}Q_{0}^{-1} \\
\text { dominant }\end{array}$ & $\frac{\omega_{0}}{\sqrt{\mu^{\prime}}}$ & $Q_{0}\left(\mu^{\prime}\right)^{3 / 4}$ & $\left(\frac{\omega_{0}}{\omega_{2}}\right)^{2}$ and $\left(\frac{Q_{2}}{Q_{0}}\right)^{4 / 3}$ & - \\
\hline $\begin{array}{c}\mu^{\prime \prime} \\
\text { dominant }\end{array}$ & $\frac{\omega_{0}}{\sqrt{\mu^{\prime}}}$ & $\frac{\mu^{\prime}}{\mu^{\prime \prime}}$ & $\left(\frac{\omega_{0}}{\omega_{2}}\right)^{2}$ & $\frac{1}{Q_{2}}\left(\frac{\omega_{0}}{\omega_{2}}\right)^{2}$ \\
\hline general & $\frac{\omega_{0}}{\sqrt{\mu^{\prime}}}$ & {$\left[\frac{1}{Q_{0}} \frac{1}{\left(\mu^{\prime}\right)^{3 / 4}}+\frac{\mu^{\prime \prime}}{\mu^{\prime}}\right]^{-1}$} & $\left(\frac{\omega_{0}}{\omega_{2}}\right)^{2}$ & $\left(\frac{\omega_{0}}{\omega_{2}}\right)^{2}\left[\frac{1}{Q_{2}}-\frac{1}{Q_{0}}\left(\frac{\omega_{2}}{\omega_{0}}\right)^{3 / 2}\right]$ \\
\hline
\end{tabular}

TABLE I. Top: Summary of the relationships between $\omega_{0}, Q_{0}, \omega_{1}, Q_{1}$ and the complex relative permittivity. Bottom: Summary of the relationships between $\omega_{0}, Q_{0}, \omega_{2}, Q_{2}$ and the complex relative permeability.

magnetic particles present to determine $\omega_{0}$ and $Q_{0}$. Next, the resonance would be remeasured with the magnetic particles suspended in the liquid to find $\omega_{2}$ and $Q_{2}$. Finally, $\mu^{\prime}$ and $\mu^{\prime \prime}$ could then be determined using the appropriate relationships given in Table $\mathbb{\amalg}$.

\section{SUMMARY}

Methods for determining the complex relative permittivities and permeabilities of materials using a SRR have been described. In general, the SRR signal response is non-Lorentzian. However, in a wide variety of cases the signal is very accurately approximated by a Lorentzian. For dielectric materials, the limiting cases of $Q_{0}^{-1} \gg \varepsilon^{\prime \prime} / \varepsilon^{\prime}$ and $Q_{0}^{-1} \ll \varepsilon^{\prime \prime} / \varepsilon^{\prime}$ as well as the general case $Q_{0}^{-1} \sim \varepsilon^{\prime \prime} / \varepsilon^{\prime}$ were all considered. Teflon, 2-propanol, and water were used as examples of these three cases and it was shown how measurements of the in-air and in-liquid resonant frequencies and quality factors can be used to experimentally determine $\varepsilon^{\prime}$ and $\varepsilon^{\prime \prime}$. An equivalent analysis for the case of a SRR submerged in a ferromagnetic suspension was also presented.
* Lake-Bobowski@ubcca; https://people.ok.ubc.ca/jbobowsk

1 D. R. Smith, W. J. Padilla, D. C. Vier, S. C. Nemat-Nasser, and S. Schultz, Phys. Rev. Lett. 84, 4184-4187 (2000).

2 D. A. Bonn, D. C. Morgan, and W. N. Hardy, Rev. Sci. Instrum. 62, 1819-1823 (1991).

${ }^{3}$ W. N. Hardy, D. A. Bonn, D. C. Morgan, R. Liang, and K. Zhang, Phys. Rev. Lett. 70, 3999-4002 (1993).

${ }^{4}$ M. Burresi, D. van Oosten, T. Kampfrath, H. Schoenmaker, R. Heideman, A. Leinse, and L. Kuipers, Science 326, 550-553 (2009).

5 J. S. Bobowski, Am. J. Phys. 81, 899-906 (2013).
${ }^{6}$ M. C. Ricci and S. M. Anlage, Appl. Phys. Lett. 88, 264102 (2006).

7 W. N. Hardy and L. A. Whitehead, Rev. Sci. Instrum. 52, 213-216 (1981).

8 The complex permittivity has been modelled using the function $\varepsilon_{\mathrm{r}}=\varepsilon_{\infty}+\left(\varepsilon_{\mathrm{s}}-\varepsilon_{\infty}\right) /(1+j \omega \tau)$.

9 F. Buckley and A. A. Marriott, Tables of Dielectric Dispersion Data for Pure Liquids and Dilute Solutions (National Bureau of Standards Circ. No. 589, 1958).

10 R. Buchner, J. Barthel, and J. Stauber, Chem. Phys. Lett. 306, 57-63 (1999). 